Radial and Nonradial Pulsations as Probes of Stellar Physics

ASP Conference Series, Vol. 259, 2002

C. Aerts, T.R. Bedding, \& J. Christensen-Dalsgaard, eds.

\title{
Pulsations in roAp Stars
}

\section{S. Cunha}

Centro de Astrofísica da Universidade do Porto, rua das Estrelas, 4150-762 Porto 8 ISMAI, Maia, Portugal, e-mail: mcunha@astro.up.pt

\begin{abstract}
The current status of the research concerning rapidly oscillating Ap stars is reviewed, paying particular attention to the interplay between recent developments in the theoretical and observational aspects of these stars.
\end{abstract}

\section{Introduction}

Rapidly oscillating Ap stars are classical Ap stars, which pulsate in high frequencies, with periods ranging from about 5 to 15 minutes. They were discovered by Kurtz (1982), and since then the number observed has increased considerably, making today a total of 32 (Martinez et al., 2001).

In the HR diagram, roAp stars are located in the main sequence part of the classical instability strip, with effective temperatures ranging between 7000 and $8500 \mathrm{~K}$. Moreover, the pulsations they exhibit are usually interpreted as high order, low degree, acoustic oscillations.

The luminosity, the magnetic field strength and the strength of the spectral lines of many Ap stars vary periodically with time. These variations were explained by Stibbs in 1950. According to Stibbs' oblique rotator model, Ap stars possess an essentially dipolar magnetic field, the axis of symmetry of which is inclined in relation to the rotation axis.

When rapid pulsations were discovered in some of the coolest Ap stars, it was realized that the corresponding light curves were also modulated on the time scale of rotation (Kurtz, 1982). Led by these observations, Kurtz introduced the oblique pulsator model, according to which each eigenmode can be described by a single spherical harmonic, with the pulsation axis aligned with the magnetic field axis. As a result of this geometry an eigenmode appears to the observer as a set of $(2 \ell+1)$ frequencies in the amplitude spectrum, each separated from the previous one by an amount that is equal to the rotation frequency.

A generalisation of the oblique pulsator model was later developed by Dziembowski \& Goode (1985), with the idea of taking into account the effects of rotation and magnetic field on the oscillations. Since new developments concerning this issue were announced in this conference, we refrain from discussing it here, and refer the readers to the paper by Bigot \& Dziembowski (these proceedings).

In the past two decades a considerable amount of data concerning roAp stars have been collected. Many new questions have been raised, and new theories have been developed in an attempt to improve our understanding of these stars. 
In the following sections we shall discuss some of these questions and some suggested answers. In Sect. 2 we discuss those issues that are related to the effect of the magnetic field on the oscillations, while in Sect. 3 we discuss the excitation mechanism and the systematic differences found between roAp stars, noAp stars (i.e. those roAp star candidates in which rapid oscillations have not been found) and Ap stars in general. Finally, in Sect. 4 we shall briefly refer to other topics concerning roAp stars, and present some conclusions.

\section{Magnetic field effects on the oscillation}

The effect of the magnetic field on the oscillations of roAp stars has been the object of several studies. It is well known that roAp stars have large-scale magnetic fields with typical intensities of the order of a few $\mathrm{kG}$ and that these fields influence both the oscillation frequencies and eigenfunctions. It is also known that correct information about the interior of these stars can only be extracted from the oscillations if their amplitude spectra are well understood.

Some of the most important recent developments concerning the study of the magnetic effect on the oscillations of roAp stars derived from the realization that in the surface layers this effect cannot be treated by standard perturbation theory. First acknowledged by Biront et al. (1982), this fact has been taken into account in many recent works discussing the magnetic perturbations to the oscillations of roAp stars (Dziembowski \& Goode, 1996; Cunha, 1999; Bigot et al., 2000; Cunha \& Gough, 2000; Cunha 2001). Even though deep enough in the interior of the star the pressure forces are much greater than the Lorentz force, near the surface the two are comparable, and a singular perturbation approach must be adopted. Accordingly, recent studies divide the star into two regions: a surface boundary layer and the interior. In these studies the magnetohydrodynamic equations with appropriate boundary conditions are solved in the surface boundary layer and the acoustic part of the corresponding solution is matched into the solution appropriate to the interior, where the magnetic field is neglected. While in the magnetic boundary layer the wave is magnetoacoustic, in the interior the magnetic and the acoustic parts decouple, giving rise to a wave whose nature is essentially acoustic, and magnetic slow waves that dissipate (Roberts \& Soward, 1983).

One important result brought about by the boundary layer approach described above concerns the shape of the perturbed eigenfunctions. The magnetic field modifies the angular shape of the eigenfunctions, causing deviation from a single spherical harmonic structure near the surface. A comparison between the magnetically perturbed and the unperturbed eigenfunctions indicates that the observer might misidentify the degree of the modes. This is particularly true for modes of larger degrees, for which the cancellation effects are more important (Dziembowski \& Goode, 1996).

That some of the eigenmodes of roAp stars may not be described by a single spherical harmonic is something that has been known for some time, from the analysis of the observations (e.g. Kurtz et al., 1989). Now that we seem to hold part of the theoretical explanation for it, the challenge is to use the observations together with the theory in order to try to extract the information that the distorted eigenfunctions may hold about the magnetic field of these stars. 


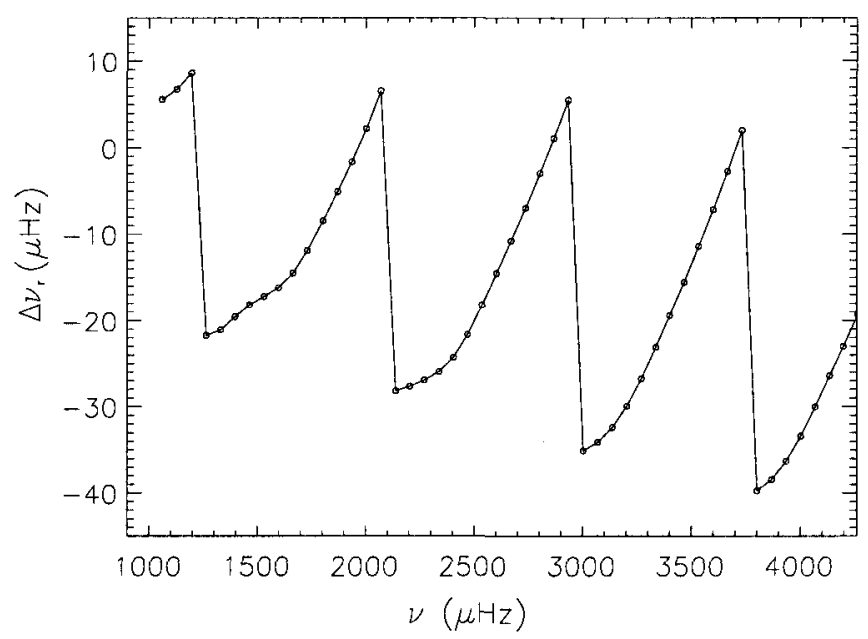

Figure 1. Real part of the magnetic perturbation to the frequencies of modes of degree $\ell=1$ and azimuthal order $m=0$ versus the unperturbed frequencies. The open circles mark the unperturbed eigenfrequencies.

Another important result brought about by the works following the boundary layer approach described above concerns the magnetic perturbation to the eigenfrequencies. According to the theoretical studies, the magnetic perturbation to the eigenfrequencies appears to be in general relatively small. However, there are frequencies at which that perturbation becomes significantly larger, and can easily lead to a wrong interpretation of the oscillation spectra. According to the work of Cunha \& Gough (2000) (see also, Cunha, 2001 and Saio, these proceedings), at certain frequencies there is an optimal coupling between the acoustic and the magnetic waves and a considerable amount of energy is transferred from the former to the latter. When such optimal coupling occurs, the magnetic perturbation to the eigenfrequencies changes abruptly, as shown in Fig. 1.

Abrupt changes in the magnetic perturbation to the eigenfrequencies may be detected observationally. It is well known that in a spherically symmetric star, high-order adiabatic acoustic oscillations are expected to be almost equally spaced in frequency (Tassoul, 1980). The presence of a magnetic field modifies the frequencies of the oscillations and therefore may affect that spacing. According to the results shown in Fig. 1, if the modes observed in a given roAp star all lie on the same side of one of these frequency "jumps", their frequencies will be modified in a similar way. Thus, the frequency spacing will be systematically larger than in the absence of a magnetic field, but by only a small amount. However, if two modes lie on opposite sides of one of these "jumps", their separation in frequency will be significantly decreased, and might be detected observationally. Based on this result, Cunha (2001) suggested that this may in fact be the 
explanation for the strange separation between the last two frequencies observed in HR 1217.

\section{What makes an Ap star become rapidly oscillating?}

\subsection{Excitation mechanism}

The issue of the excitation mechanism responsible for the oscillations observed in roAp stars has been debated since these pulsators were first discovered. Several ideas concerning the excitation mechanism were proposed over the years (Dolez \& Gough, 1982; Dolez et al., 1988; Shibahashi, 1983; Dziembowski, 1984; Dziembowski \& Goode, 1985; Matthews, 1988). However, some of these ideas have not been pursued while some others have failed to show that models appropriate to roAp stars should be unstable to high frequency oscillations.

More recently, two groups claimed to have found models that may be appropriate to roAp stars and which are unstable to high frequency oscillations (Gautschy et al., 1998, Balmforth et al., 2001). In both works the oscillations are found to be excited primarily in the region of hydrogen ionization. Before discussing these two recent works we would like to emphasise that the problem of the excitation mechanism in roAp stars cannot be looked at without considering, simultaneously, the problem of the acoustic cutoff frequency. In fact, no matter how efficient the excitation mechanism is, if the waves are not reflected as they travel towards the surface of the star, they will eventually dissipate. In roAp stars the process of reflection of the acoustic waves near the surface is still not well understood. However, since this issue has been extensively discussed in a previous review (Cunha, 1998), we will refrain from discussing it further here, referring to it only where necessary.

The models developed by Gautschy et al. (1998) differ from previous models of roAp stars mainly in their atmospheric structure. Considering the possibility of roAp stars having a chromosphere of some extent, Gautschy et al. modified a temperature-optical depth $(T-\tau)$ relation originally fitted to a model atmosphere of Kurucz in such a way as to induce a temperature inversion at small optical depths. This temperature inversion helps the reflection of the high frequency modes and contributes to destabilize them. While illustrating well the fact that the reflection of the waves near the surface is an essential problem when considering the excitation mechanism in roAp stars, the present work assumes the presence of a chromosphere for which there is no observational evidence (e.g. Shore et al., 1987). This fact is acknowledged by the authors and should be taken as a motivation for the continued searching for a possible signature of chromospheric activity in roAp stars.

The models developed by Balmforth et al. (2001) differ from previous models of roAp stars mainly in their surface convective layers. Bearing in mind that roAp stars have large scale, relatively strong, magnetic fields, the authors argue that convection might be suppressed in the magnetic polar regions of these stars. Accordingly, their models are composed of two polar regions, in which envelope convection is totally suppressed, and an equatorial region, where convection is unaffected. Believing that the surface reflection of the oscillations in roAp stars is an issue that cannot be solved without taking into account the magnetoacoustic nature of the waves (e.g. Cunha, 1998), the authors did not attempt to reproduce 
the unknown reflection process, but rather decided to test the robustness of their results by applying different boundary conditions. In an attempt to bracket the physically plausible range of atmospheric conditions, the authors computed pulsational stability with two different outer mechanical boundary conditions, the first of which is perfectly reflective. The second boundary condition is that appropriate to a plane-parallel isothermal atmosphere whose temperature matches continuously with that of the underlying envelope, thus representing a star with no chromosphere. In both cases Balmforth et al. found high frequency unstable modes in models with temperatures and luminosities typical of roAp stars.

\section{2. roAp stars versus noAp stars and Ap stars in general}

One of the challenges that any theory concerning the excitation mechanism in roAp stars has to face is the fact that many candidate roAp stars have been found not to pulsate. Understanding what are the main differences between the latter, often called non oscillating Ap stars (or, noAp stars), and the roAp stars may be an important step to the understanding of the excitation process. Unfortunately, it is impossible to know whether noAp stars are stable to high frequency pulsations, or whether their oscillations have been systematically missed by the observations. Therefore, both these possibilities have to be kept in mind.

Different efforts have been made in the past years with the aim of establishing the observational differences between roAp and noAp stars. Concerning their position in the HR diagram, using the Hipparcos parallaxes for sets of roAp and noAp stars, North et al. (1997) and Hubrig et al. (2000) have concluded that noAp stars are systematically more luminous and more evolved than roAp stars.

Bearing in mind that rapid oscillations might have been systematically missed in noAp stars, Hubrig et al. discussed the possibility of the differences found in their work being the result of an observational bias. According to the authors, noAp stars are systematically fainter, in apparent magnitudes, than roAp stars. Therefore, it is possible that the oscillations that they may exhibit have been missed in the observations. This, together with the fact that noAp stars are further away on average and are more numerous than roAp stars, having a greater chance of spanning the whole width of the main sequence, could, as argued by the authors, make noAp stars look intrinsically brighter than roAp stars. Another possible observational bias was suggested by Cunha (2000), based on theoretical results obtained with models similar to those used by Balmforth et al. (2001). According to Cunha (2000), the frequencies of the unstable modes found in models appropriate to roAp stars decrease with increasing luminosity and with decreasing effective temperature. Therefore, stars that are more luminous and/or more evolved might tend to pulsate in lower frequencies. Given that the sky transparency can become a serious problem when observing oscillations with frequencies just below $1 \mathrm{mHz}$, the author argues that the oscillations in these more evolved and/or more luminous stars, having smaller frequencies, might be missed more often. Naturally, satellites like MOST, MONS, COROT and Eddington are in a privileged position to clarify this matter.

Even though biases like those referred above might have affected the statistical analysis carried out by Hubrig et al. (2000), the systematic differences found in that analysis may have their origin in intrinsic differences between these two types of stars. If that is the case, it is the job of the theories of mode ex- 
citation to explain why some cool Ap stars become unstable to high frequency oscillations while others, with similar characteristics, remain stable. Concerning this possibility, one may argue (Cunha, in preparation), based on models similar to those used by Balmforth et al. (2001), that the suppression of convection by the magnetic field is less likely to happen when the stars are more evolved. If the suppression of convection is essential for the excitation of high frequency oscillations in roAp stars, as suggested by Balmforth et al. (2001), this result might help to explain why noAp stars are systematically more evolved. Moreover, the predicted growth rates for the unstable modes decrease as the star evolves, and, therefore, modes might be more easily stabilized in more evolved stars by any additional source of energy loss (like the coupling with the Alfvén waves) not included in the calculations.

Another important difference that has been found between roAp and noAp stars concerns their surface chemical composition (Ryabchikova et al., 2000 and references therein). In fact, spectroscopic studies of the atmospheres of Ap stars have indicated a difference in the relative abundance of the first and second ions of some rare earth elements in roAp and noAp stars. More details on these observational differences are discussed by Weiss et al. (these proceedings). From the theoretical point of view, we would just like to stress that while no clear explanation has yet been given for the origin of these differences, it is likely that differences in the atmospheric structure of these two types of stars will produce differences in their surface abundances. Therefore, it would be interesting to consider diffusion in model atmospheres modified to match the suggestions of Gautschy et al. (1998) and of Balmforth et al. (2001), in order to investigate whether either of these could explain the referred observations.

Finally, some systematic differences seem to have been found between roAp stars and Ap stars in general. These include differences related to the magnetic field (Mathys et al., 1997) and the absence of spectroscopic binaries among roAp stars (e.g. Hubrig \& Mathys, 2000). In particular, Mathys et al. point out that the fraction of stars with magnetically resolved lines is significantly higher among roAp stars than among Ap stars in general. Even though the significance of this result is not very clear, due to a bias in the selection of the targets, if significant this result may be of great importance for the understanding of the conditions necessary for the excitation of high frequency oscillations in cool Ap stars.

\section{Other exciting topics and conclusions}

It is impossible, in a review like this one, to describe in any depth all the work that has been carried out concerning roAp stars in recent years. For that reason we have chosen to concentrate our attention on a few issues that seemed of relevance to the subject. However, it seems to us that other exciting topics, that have been developed recently but not mentioned above, should at least be mentioned as we conclude, as a motivation for further reading.

Of particular interest are the multicolour photometric observations and the spectroscopic observations aiming at determining the radial structure of the eigenfunctions (e.g. Kurtz \& Medupe, 1996; Medupe \& Kurtz, 1998; Baldry et al., 1999; Baldry \& Bedding, 2000, see also Kochukhov \& Ryabchikova, these proceedings). As mentioned above, the magnetic field, among others, influences 
the structure of the eigenfunctions in the surface layers. Therefore, if the shape of the eigenfunctions is determined observationally one might hope to use it together with the theory, in order to learn about the magnetic field geometry and intensity.

Also important is that excitation theories are continuously tested against new observations like the northern hemisphere surveys that have been started recently (Handler \& Pauzen, 1999; Martinez et al., 2001). Also, targets appropriate to test these theories should, when possible, be included in the new surveys.

The high frequencies of the oscillations observed in roAp stars make this type of pulsators particularly suited for asteroseismology. Attempts to learn more about these stars from the frequencies of their oscillations have been carried out (e.g. Matthews et al., 1999), which have raised interesting new questions. Definitely, the answers to these questions should be pursued.

Finally, it is important that observational efforts aimed at learning about the properties of the atmospheres of roAp stars are continued, and that their results are used to improve the theoretical atmospheric models of roAp stars.

Acknowledgments. Margarida S. Cunha is supported by the Portuguese Fundação para a Ciência e a Tecnologia through the grant PD/18893/98.

\section{References}

Baldry, I.K., et al. 1999, MNRAS, 302, 381

Baldry, I.K. \& Bedding, T. R. 2000, MNRAS, 318, 341

Balmforth, N. J. et al. 2001, MNRAS, 323, 362

Bigot, L., et al. 2000, A\&A, 356, 218

Biront, D., et al. 1982, MNRAS, 201, 619

Cunha, M. S. 1998, Contrib. Astron. Obs. Skalnaté Pleso, 27, 272

Cunha, M. S. 1999, PhD Thesis, Cambridge University, UK

Cunha, M. S. \& Gough, D. O. 2000, MNRAS, 319, 1020

Cunha, M. S., 2000, in Proceedings of the 3th MONS Workshop, eds. T.R. Bedding \& T.C. Teixeira, Aarhus, Denmark

Cunha, M. S. 2001, MNRAS, 325, 373

Dolez, N. \& Gough, D.O. 1982, in Pulsations in Classical and Cataclysmic Variable stars, eds. J. P. Cox \& C.J. Hansen (JILA, Bouder, CO), 248

Dolez, N., Gough, D.O., \& Vauclair, S. 1988, in Advances in Helio- and Asteroseismology, eds. J. Christensen-Dalsgaard \& S. Frandsen, 291

Dziembowski, W. A. 1984, in Theoretical problems in stellar stability and oscillations, eds. M. Gabriels \& A. Noels (Université de Liège), 346

Dziembowski, W. A. \& Goode, P. R. 1985, ApJ, 296, L27

Dziembowski, W. A. \& Goode, P. R. 1996, ApJ, 458, 338

Gautschy, A., Saio, H. \& Harzenmoser, H. 1998, MNRAS, 301, 31

Handler, G. \& Paunzen, E. 1999, A\&AS, 135, 57

Hubrig, S., et al., 2000, A\&A, 355, 1031 
Kurtz, D. W. 1982, MNRAS, 200, 807

Kurtz, D. W., et al. 1989, MNRAS, 240, 881

Kurtz, D. W. \& Medupe, R. 1996, BASI, 24, 291

Martinez, P., et al. 2001, A\&A, 371, 1048

Mathys, G., et al. 1997, A\&AS, 123, 353

Matthews, J. M. 1988, MNRAS, 235, 7

Matthews, J. M., Kurtz, D. W. \& Martinez, P. 1999, ApJ, 511, 422

Medupe, R. \& Kurtz, D. W. 1998, MNRAS, 299, 371

North, P., et al. 1997, in Hipparcos-Venice 97, (ESA SP- 402), 239

Roberts, P. H. \& Soward, A. 1983, MNRAS, 205, 1171

Ryabchikova, T.A., et al. 2000, A\&A, 357, 981

Shibahashi, H. 1983, ApJ, 275, L5

Shore, S.N., et al. 1987, A\&A, 182, 285

Stibbs, D.W.N. 1950, MNRAS, 110, 395

Tassoul, M. 1980, ApJS, 43, 469

\section{Discussion}

N. Evans : I recall, not only are there relatively few Ap stars in binaries, they only exist in relatively wide spectroscopic binaries. Can you confirm this, and, does it point to information about the creation and/or the destruction of magnetic fields?

M. Cunha: There are discussions as to whether it is binarity that influences the magnetic fields in Ap stars or whether it is the other way around. If the result mentioned by Mathys et al. (1997) about the larger incidence of stars with magnetically resolved lines among roAp stars is significant, and if we believe that the suppression of convection is needed for the excitation of high frequency oscillations in these stars, then one is tempted to speculate that the magnetic field might be systematically larger in roAp stars than among Ap stars in general. This, in turn, would clearly support the idea that there is an interplay between binarity and magnetic field. Whether it would solve the question of which influences which, is something that should be investigated further.

$M$. Goossens : Can you comment on the locking of the pulsation axis to the magnetic axis?

M. Cunha : In roAp stars the rotation axis and the magnetic field axis are not aligned. Thus, the axis of pulsation is neither the magnetic field axis, nor the rotation axis. Whether it is closer to the former or to the latter, depends on the relative intensity of the magnetic and rotational effects. If only the coriolis force it taken into account, the magnetic effect is expected to largely dominate the effect of rotation and to first approximation the pulsation axis is expected to be locked to the magnetic axis. However, Bigot et al. (these proceedings) have shown that the effect of the centrifugal force cannot be neglected in roAp stars and thus the conclusions concerning the alignment between pulsation and magnetic axis may have to be reviewed. 